Case report

\title{
Therapeutic effect of hyperbaric oxygen in psoriasis vulgaris: two case reports and a review of the literature Glenn Butler ${ }^{1 *}$, Julio Chávarri Michaels ${ }^{5}$, Noori Al-Waili ${ }^{1,2}$, Michael Finkelstein ${ }^{3}$, Michael Allen ${ }^{1}$, Richard Petrillo ${ }^{2}$, Zev Carrey ${ }^{2}$, Bangaruraju Kolanuvada ${ }^{2}$, Bok Y Lee ${ }^{1,4}$, Alfonso Gonzales Riera ${ }^{5}$, Cesar Chávarri Michaels ${ }^{5}$ and Gary Urteaga ${ }^{6}$
}

\footnotetext{
Addresses: ${ }^{1}$ Life Support Technologies, The Mount Vernon Hospital, Mount Vernon, NY, USA

${ }^{2}$ Department of Medicine, The Mount Vernon Hospital, South Shore Health System, Valley Stream, NY, USA

${ }^{3}$ Dr J Beale's Chronic Wound Management and Hyperbaric Center, The Mount Vernon Hospital, Mount Vernon, NY, USA

${ }^{4}$ Department of Surgery, New York Medical College, New York, USA

${ }^{5}$ Lima Center for Hyperbaric Medicine, Peru

${ }^{6}$ Corporación Hiperbárica Peruana, Peru

Email: GB* - gjblst@aol.com; JCM - gjblst@aol.com; NAW - drnoori6@yahoo.com; MF - gjblst@aol.com; MA - gjblst@aol.com; RP - zavmd@aol.com; ZC - zavmd@aol.com; BK - BYLee2100@aol.com; BL - zavmd@aol.com; AGR - gjblst@aol.com; CCM - gjblst@aol.com; GU - gjblst@aol.com

* Corresponding author
}

Received: 30 July 2008 Accepted: 23 January 2009 Published: 10 August 2009

Journal of Medical Case Reports 2009, 3:7023 doi: 10.4076/1752-1947-3-7023

This article is available from: http://jmedicalcasereports.com/jmedicalcasereports/article/view/7023

(C) 2009 Butler et al.; licensee Cases Network Ltd.

This is an Open Access article distributed under the terms of the Creative Commons Attribution License (http://creativecommons.org/licenses/by/3.0), which permits unrestricted use, distribution, and reproduction in any medium, provided the original work is properly cited.

\begin{abstract}
Introduction: Psoriasis is an inflammatory and immunological cutaneous disease. The high morbidity in patients with psoriasis results from severe clinical manifestations and/or adverse effects of treatment. The Undersea and Hyperbaric Medical Society and Federal Medicare and Medicaid Services have approved the use of hyperbaric oxygen $\left(\mathrm{HBO}_{2}\right)$ for more than 15 indications, including wound healing, infections and late effects of radiation, which are largely unresponsive to conventional treatments. Accumulated data show that $\mathrm{HBO}_{2}$ has anti-inflammatory effects and other positive influences on the immune system, making it a rational treatment in the management of psoriasis plaques and arthritis.
\end{abstract}

Case presentation: We present the cases of two patients with long histories of psoriasis vulgarus who exhibited marked improvement with use of $\mathrm{HBO}_{2}$. The first patient was 40 years old and had pustular psoriasis and psoriatic arthritis. He was treated with six sessions of $\mathrm{HBO}_{2}$ (at 2.8 atmospheres of pressure for 60 minutes), which successfully controlled his symptoms. At the 18month post-treatment follow up, the patient exhibited complete remission of psoriasis and marked improvement in psoriatic arthritis without medication. The second patient was 55 years old with extensive psoriatic lesions, and exhibited marked improvement within 15 sessions of $\mathrm{HBO}_{2}$. No adverse effects of $\mathrm{HBO}_{2}$ were identified.

Conclusions: $\mathrm{HBO}_{2}$ may possess potential therapeutic efficacy in the management of psoriasis. We outline the pathogenesis of psoriasis and the selective anti-inflammatory and immunosuppressive effects of $\mathrm{HBO}_{2}$. We hope that this will provide a basis for elucidating the mechanisms of action and consequently pave the way for further controlled studies. 


\section{Introduction}

Psoriasis is a chronic, remitting and relapsing, immunemediated inflammatory skin disorder with a strong genetic predisposition. It is among the most common immunemediated diseases in humans, affecting $2.6 \%$ of the US population, and has significant social and economic impact. Current topical therapies used to manage psoriasis include steroids, vitamin $\mathrm{D}$ derivatives, retinoids, immunosuppressants, anthralin, coal tar ointment, and several other agents [1-5]. These drugs often have adverse effects that may be poorly tolerated. Light therapy includes ultraviolet B phototherapy or psoralen and ultraviolet A (PUVA) photochemotherapy. However, increased rates of nonmelanoma skin cancer have been observed following PUVA therapy [6]. Systemic therapies for psoriasis include methotrexate, cyclosporine, oral retinoids, and biologic therapies. A recent report reviewed the effectiveness and safety of the biologics alefacept, efalizumab, etanercept, and infliximab [7]. In addition to the reported adverse effects of the drugs, it was found that up to $40 \%$ of patients did not use their medication as directed.

Hyperbaric oxygen $\left(\mathrm{HBO}_{2}\right)$ treatment is defined as breathing pure $(100 \%)$ oxygen under conditions of increased atmospheric pressure. This results in elevated arterial oxygen tension to $2,000 \mathrm{mmHg}$ or greater, which provides tissues with abundant oxygen. Possible complications of $\mathrm{HBO}_{2}$ therapy include barotrauma, oxygen toxicity (affecting the central nervous system and lungs), claustrophobia and anxiety, and ocular effects such as myopia and cataract. $\mathrm{HBO}_{2}$ promotes proliferation of fibroblasts, epithelial cells, and blood vessels in a wound. It can increase the killing ability of leukocytes and is lethal to certain anaerobic bacteria. Furthermore, it inhibits toxin formation by certain anaerobes, increases the flexibility of red cells, reduces tissue edema, and conserves intracellular ATP.

The Undersea and Hyperbaric Medical Society and the Federal Center for Medicare and Medicaid Services have approved the use of $\mathrm{HBO}_{2}$ in 14 indications including gas gangrene, necrotizing soft-tissue infections, diabetic foot ulcer, compromised grafts and flaps, bone infection, intracranial abscess, anemia and blood loss, crush injury, carbon monoxide and cyanide poisoning, radiation complications, decompression sickness, and gas embolism. $\mathrm{HBO}_{2}$ has potential effects on mediators of inflammation and the immune response. Our recent reviews $[8,9]$ support the contention that $\mathrm{HBO}_{2}$ has antiinflammatory and immunosuppressive properties. These properties make this treatment a potentially useful intervention that should be tested in the management of psoriasis and psoriatic arthritis.

\section{Case presentation \\ Case I}

A 40-year-old man with disseminated erythrodermic psoriasis with pustules presented with arthralgias. He had a history of psoriasis vulgaris from infancy, diagnosed by skin biopsy, and had been followed by dermatologists. He had also had psoriatic arthritis since childhood. Physical examination revealed erythematous scaly plaques on his elbows, trunk and umbilical area, perineum and legs (Figure 1). He had interphalangeal distal joint involvement and spondylitis. His medical history was uneventful, with no evidence of underlying systemic disease and he was not taking any medications at the time of presentation.

He requested a hyperbaric consultation after reading a newspaper article reporting on the use of $\mathrm{HBO}_{2}$ to treat psoriasis in a study conducted in Cuba. At the hospital he was evaluated to determine whether he was a suitable candidate for $\mathrm{HBO}_{2}$ therapy. After he had given informed consent, he underwent $\mathrm{HBO}_{2}$ therapy at 2.8 atmospheres for 60 minutes, once a day ( 5 days per week). The patient underwent a total of eight sessions, resulting in significant amelioration of symptoms (Figure 2). The patient did not receive any topical treatment before or during $\mathrm{HBO}_{2}$ therapy.

Most of the psoriatic lesions were cleared, with marked reduction in itching and scaling. No side effect was reported with the use of $\mathrm{HBO}_{2}$. The patient also reported less pain in his hands and joints after the eight sessions of $\mathrm{HBO}_{2}$. Follow up at 18 months revealed that the patient had only mild skin symptoms with degenerative changes of the arthritis.

\section{Case 2}

A 55-year-old man was referred for $\mathrm{HBO}_{2}$ for management of chronic bilateral leg ulcers and osteomyelitis. In addition, he had extensive psoriasis vulgaris (Figure 3). He had a long history of psoriasis vulgaris, which had been diagnosed by skin biopsy and followed by a dermatologist. His current medications at the time of admission to the hyperbaric department were topical mineral oil, Eucerin Calming Creme, and diphenhydramine 50 three times a day. He had erythema, and his skin was scaling and itching.

He underwent daily $\mathrm{HBO}_{2}$ at 2 atmospheres for 90 minutes, once a day (5 days per week). After six sessions his erythema, scaling and itching were reduced in severity, and after 15 sessions he had improved further (Figure 4). No adverse effect was reported with the use of $\mathrm{HBO}_{2}$.

\section{Discussion}

The results presented here demonstrate the effectiveness of $\mathrm{HBO}_{2}$ in alleviating signs and symptoms of psoriasis in 


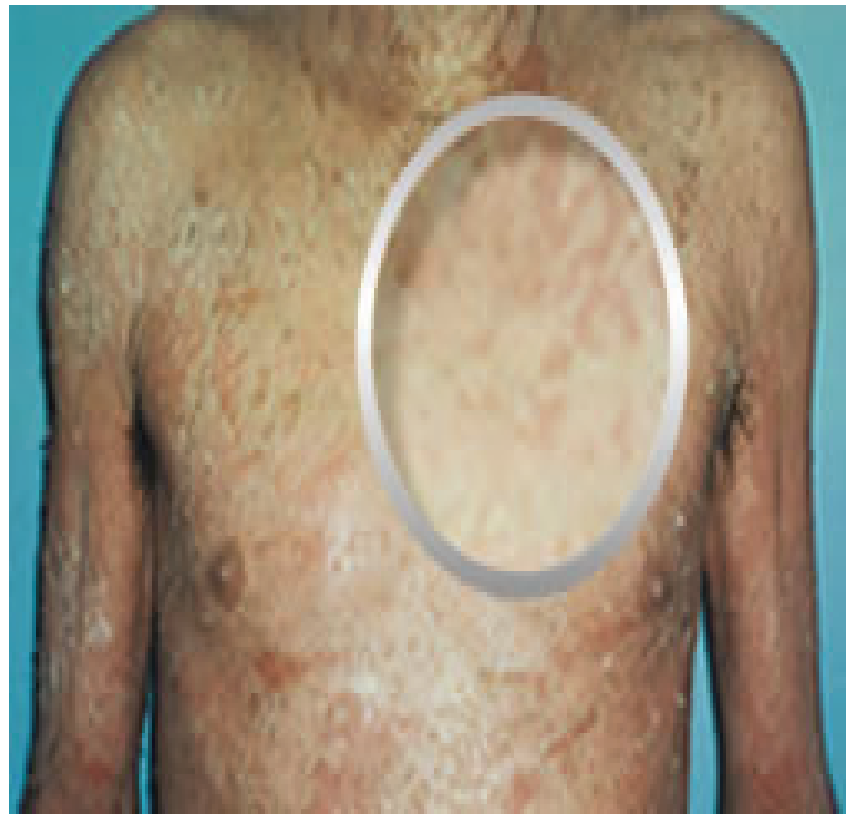

Figure I. Patient I before treatment with hyperbaric oxygen (Front View).

two patients. No adverse effects were reported during or after treatment with $\mathrm{HBO}_{2}$.

Leukocytes, cytokines, and keratinocyte growth or differentiation abnormalities are involved in psoriatic skin lesions. Psoriasis vulgaris is a T-cell-driven disease, with type I (interferon- $\mathrm{y}$-producing) $\mathrm{T}$ cells predominating in skin lesions [10,11]. A lymphocytic infiltrate in psoriasis plaques consists of a mixture of activated $\mathrm{CD} 4^{+}$and $\mathrm{CD} 8^{+}$ $\mathrm{T}$ cells; the latter predominate in lesional epidermis and $\mathrm{CD}^{+}$cells in the dermis [12]. The therapeutic benefit of

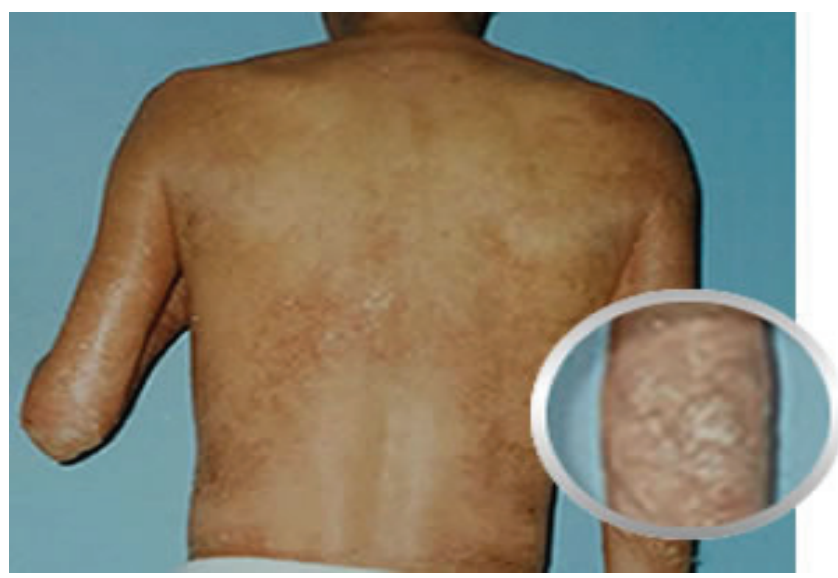

Figure 3. Patient I before treatment with hyperbaric oxygen (Back View).

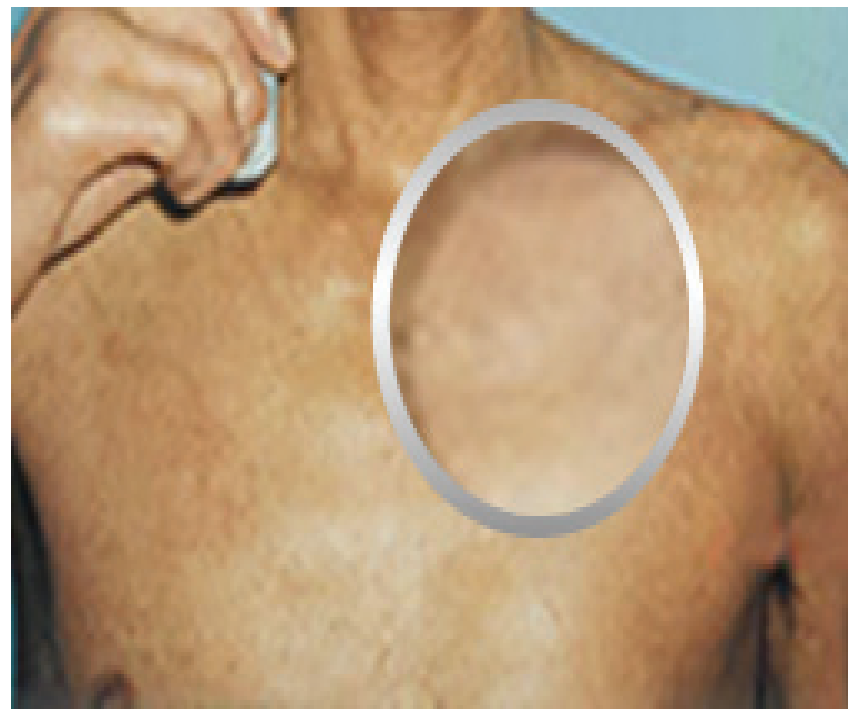

Figure 2. Patient I after treatment with hyperbaric oxygen (Front View).

immunosuppressive drugs supports the view that activated T cells are pathogenic effectors of psoriasis [10]. Dendritic cells are found in psoriatic skin lesions, producing interleukin (IL)-12 and IL-23. Cytokine changes in psoriatic lesions consist of elevated levels of interferon- $\gamma$, tumor necrosis factor (TNF)- $\alpha$, numerous interleukins (such as IL-1, IL-2, IL-6, IL-8, IL-12, IL-17, and IL-19), and multiple chemokines (MIG/CXCL9, IP-10/CXCL10, I-TAC/CXCL11, and MIP3 $\alpha / C C L 20)$ [11]. IL-12 p40 mRNA and expression of interferon- $\gamma$, inducible nitric

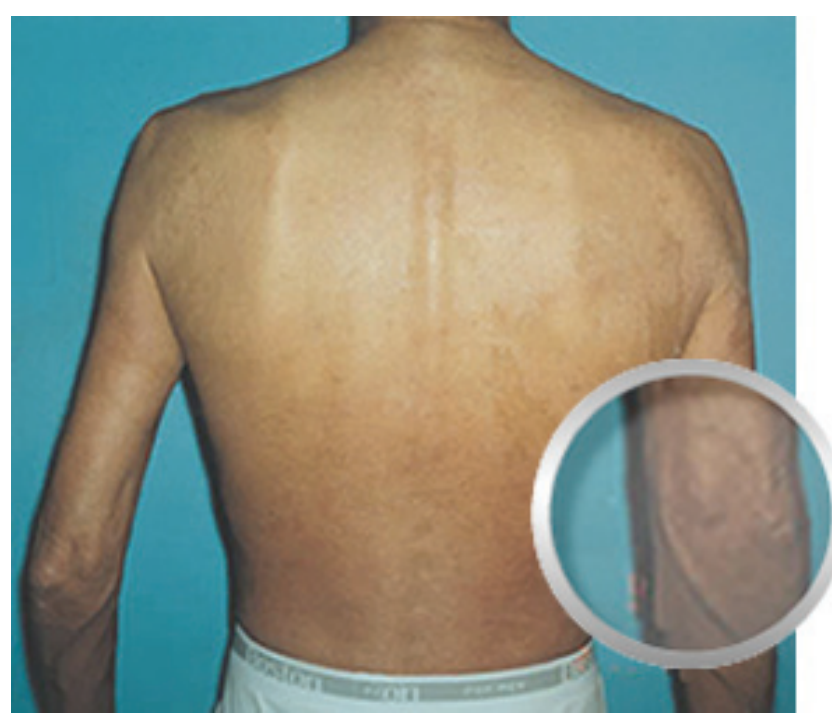

Figure 4. Patient I after treatment with hyperbaric oxygen (Back View). 


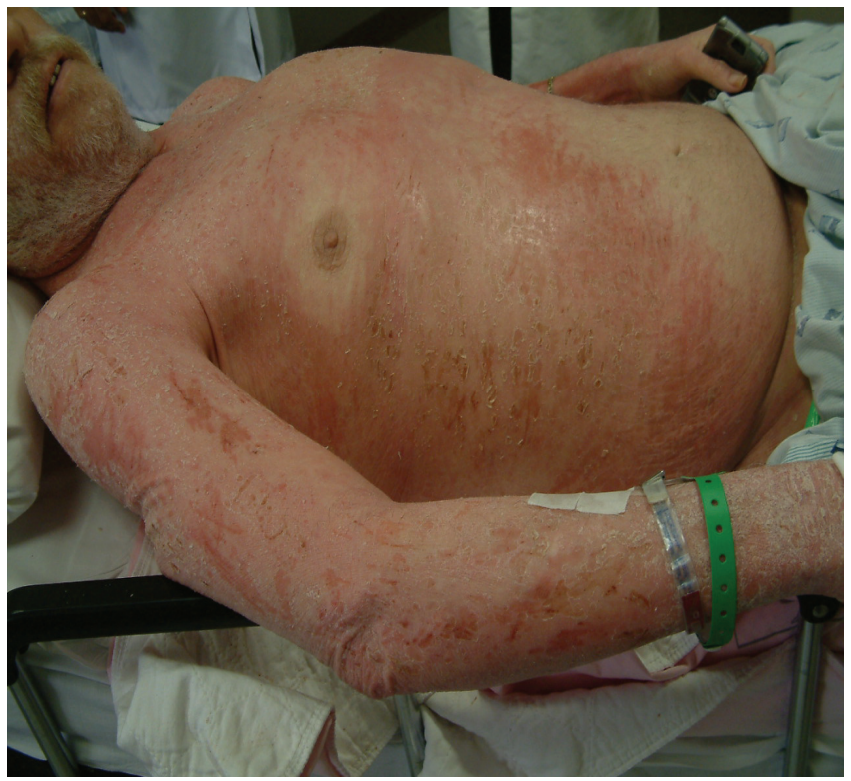

Figure 5. Patient 2 before treatment with hyperbaric oxygen (Side View Torso).

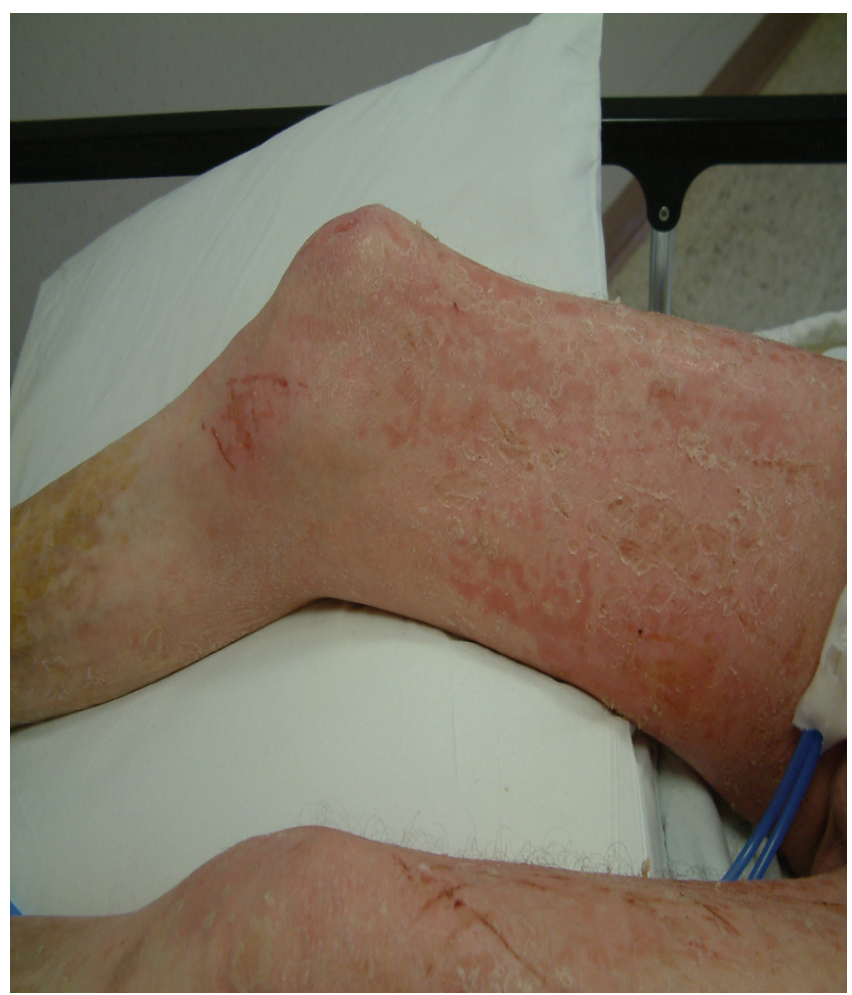

Figure 7. Patient 2 before treatment with hyperbaric oxygen (Side View Legs).

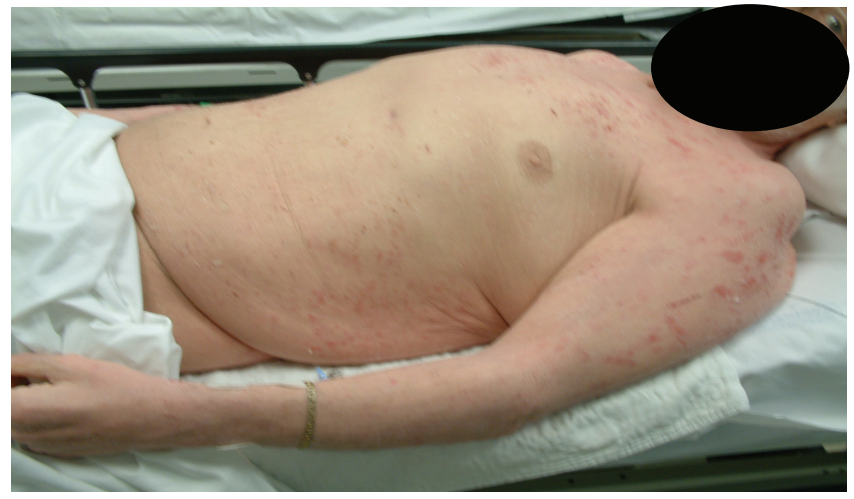

Figure 6. Patient 2 after treatment with hyperbaric oxygen (Side View Torso).

oxide synthase, B7-1, and TNF- $\alpha$ are elevated in psoriatic tissue [11]. A rheumatoid-like pattern has been identified as one of the most common types of psoriatic arthritis. Autoantibodies directed against nuclear antigens, cytokeratins, epidermal keratins, and heat shock proteins have also been reported in psoriatic arthritis.

$\mathrm{HBO}_{2}$ suppresses the proliferation of macrophages and the formation of foam cells in atherosclerotic lesions [12]. $\mathrm{HBO}_{2}$ also intensifies the suppressive function of $\mathrm{T}$ lymphocytes, normalizes cell-bound immunity, and decreases the serum concentration in immune complexes [13]. The immunosuppressive effects of $\mathrm{HBO}_{2}$ include suppression of autoimmune symptoms, decreased production of IL- 1 and $\mathrm{CD}^{+}$cells, and increased percentage and absolute number of $\mathrm{CD}^{+}$cells [9]. In addition, longterm $\mathrm{HBO}_{2}$ exposure suppresses development of autoimmune symptoms such as proteinuria, facial erythema,

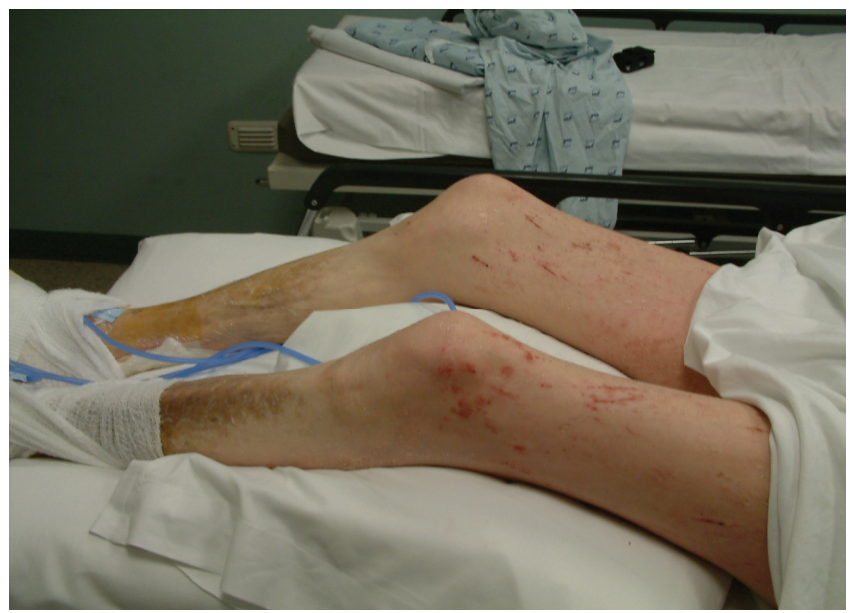

Figure 8. Patient 2 after treatment with hyperbaric oxygen (Side View Legs). 
and lymphadenopathy. $\mathrm{HBO}_{2}$ decreases the $\mathrm{CD} 4: \mathrm{CD} 8$ ratio and proliferation of lymphocytes, and activates neutrophils to migrate to regions of high oxygen tension [14]. $\mathrm{HBO}_{2}$ suppresses TNF- $\alpha$ production induced by lipopolysaccharide, lipid $\mathrm{A}$, and phytohemagglutinin $\mathrm{A}$ [15]. A marked decrease in IL-1 and IL-2 production, and a significant decrease in prostaglandin $\mathrm{E}_{2}$ production have been observed.

The positive clinical effects that $\mathrm{HBO}_{2}$ has in the treatment of chronic inflammation may relate to its effects on secretion of IL-1, IL-6, and TNF- $\alpha$. The effects of $\mathrm{HBO}_{2}$ on prostaglandin, nitric oxide, and cytokines involved in wound pathophysiology and inflammation in particular were recently reviewed [8]. That review indicates that $\mathrm{HBO}_{2}$ has important effects on the biology of cytokines and other mediators of inflammation. $\mathrm{HBO}_{2}$ causes downregulation of cytokines and upregulation of growth factors. It transiently suppresses stimulus-induced proinflammatory cytokine production and affects the liberation of TNF- $\alpha$ and endothelins. Vascular endothelial growth factor levels are significantly increased with $\mathrm{HBO}_{2}$ therapy, whereas levels of prostaglandin $\mathrm{E}_{2}$ and cyclo-oxygenase-2 mRNA are markedly reduced. Therefore, the anti-inflammatory and immunosuppressive properties of $\mathrm{HBO}_{2}$ might account for its efficacy in the cases presented here.

\section{Conclusions}

Our case reports, although suggestive, do not allow one to conclude that $\mathrm{HBO}_{2}$ treatment is useful in the treatment of psoriasis, because this condition can improve spontaneously. We emphasize that the findings presented here require confirmation by further controlled studies before a definitive conclusion may be drawn. We hope that our findings will also stimulate further investigation of the therapeutic potential of $\mathrm{HBO}_{2}$ alone or in combination with other modalities such as phototherapy in psoriasis.

$\mathrm{HBO}_{2}$ therapy may have a place in the management of psoriasis. Further studies including large numbers of patients and involving monitoring cytokines and inflammatory mediators will help us to explore the effect of hyperoxygenation on psoriasis and to elucidate its mechanism of action.

\section{Abbreviations}

$\mathrm{HBO}_{2}$, hyperbaric oxygen; IL, interleukin; PUVA, psoralen and ultraviolet A; TNF, tumor necrosis factor.

\section{Competing interests}

The authors declare that they have no competing interests.

\section{Consent}

Written informed consent was obtained from the patient for publication of this case report and any accompanying images. A copy of the written consent is available for review by the Editor-in-Chief of this journal.

\section{Authors' contributions}

GB conceived of the report and participated in its writing. NA-W conceived of the report, and conducted work and writing. $\mathrm{RP}, \mathrm{ZC}, \mathrm{BK}, \mathrm{MF}, \mathrm{MA}$, and $\mathrm{BL}$ were involved in writing the Discussion. JM conceived of the report and conducted work. GU conceived of the report and conducted work. CM conceived of the report and conducted work. AR conceived of the report and conducted work. All authors read and approved the final manuscript.

\section{References}

I. Mason J, Mason AR, Cork MJ: Topical preparations for the treatment of psoriasis: a systematic review. Br J Dermatol 2002 , | 46:35|-364.

2. Ashcroft DM, Po AL, Williams HC, Griffiths CE: Systematic review of comparative efficacy and tolerability of calcipotriol in treating chronic plaque psoriasis. BMJ 2000, 320:963-967.

3. Weinstein GD, Koo JY, Krueger GG, Lebwohl MG, Lowe NJ, Menter MA: Tazarotene cream in the treatment of psoriasis: two multicenter, double-blind, randomized, vehicle-controlled studies of the safety and efficacy of tazarotene creams $0.05 \%$ and $0.1 \%$ applied once daily for 12 weeks. J Am Acad Dermatol 2003, 48:760-767.

4. Lebwohl MG, Breneman DL, Goffe BS, Grossman JR, Ling MR, Milbauer J: Tazarotene $\mathbf{0 . 1 \%}$ gel plus corticosteroid cream in the treatment of plaque psoriasis. I Am Acad Dermatol 1998, 39:590-596.

5. Bruner $C R$, Feldman SR, Ventrapragada $M$, Fleischer $A B$ Jr: $\mathbf{A}$ systematic review of adverse effects associated with topical treatments for psoriasis. Dermatol Online J 2003, 9:2.

6. Naldi L, Rzany B: Chronic plaque psoriasis. Clin Evid 2004, I I:2 |40-2 | 67.

7. Boehncke WH, Prinz J, Gottlieb $A B$ : Biologic therapies for psoriasis. A systematic review. J Rheumatol 2006, 33:|447-|45I.

8. Al-Waili N, Butler G: Effects of hyperbaric oxygen on inflammatory response to wound and trauma: possible mechanism of action. ScientificWorldjournal 2006, 6:425-432.

9. Al-Waili N, Butler G, Petrillo RL, Carrey Z, Hamilton RW: Hyperbaric oxygen and lymphoid system function: a review supporting possible intervention in tissue transplantation. Technol Health Care 2006, 14:489-498.

10. Gottlieb S, Gilleaudeau P, Johnson R, Estes L, Woodworth TG, Gottlieb $A B$ : Response of psoriasis to a lymphocyte-selective toxin (DAB389IL-2) suggests a primary immune, but not keratinocyte, pathogenic basis. Nat Med 1995, I:442-446.

II. Krueger J: The immunologic basis for the treatment of psoriasis with new biologic agents. J Am Acad Dermatol 2002, 46: 1 -23.

12. Kudchodkar B, Wilson J, Lacko A, Dory L: Hyperbaric oxygen reduces the progression and accelerates the regression of atherosclerosis in rabbits. Arterioscler Thromb Vasc Biol 2000, 20:1637-1643.

13. Lukich V, Poliakova L, Sotnikova T, Belokrinitskii D: Hyperbaric oxygenation in the comprehensive therapy of patients with rheumatoid arthritis (clinico-immunologic study) [in Russian]. Fiziol Zh 1991, 37:55-60.

14. Brenner I, Shephard R, Shek P: Immune function in hyperbaric environments, diving, and decompression. Undersea Hyperb Med 1999, 26:27-39.

15. Benson M, Minter M, Osborne B, Granowitz E: Hyperbaric oxygen inhibits stimulus-induced proinflammatory cytokine synthesis by human blood-derived monocyte-macrophages. Liver Transpl Surg 2003, 134:57-62. 